\title{
Low-Frequency Shelf/Slope Responses Induced by Localized Offshore Forcings
}

\author{
Bo QIU ${ }^{1}$ \\ Geophysical Institute, Kyoto University, Kyoto, Japan
}

\begin{abstract}
Using a two-layer $\beta$ plane model with bottom friction, we investigated the shelf/slope responses induced by localized offshore forcing sources which (1) propagate in the along-slope direction and (2) have oscillating amplitudes. The two cases represent typical forcing patterns of cutoff eddy propagations and unstable meanders in western boundary currents. When the localized forcing source propagates in the along-slope direction, it tends to induce components of bottom-trapped topographic Rossby waves (TRWs) with a similar cross-slope structure, and the shelf/slope response depends strongly upon the magnitude of the propagating speed $c$. In the inviscid limit, maximum response occurs when $c$ is such that the induced bottom-trapped TRW components may freely cross the slope region. When bottom friction is considered, however, the shelf/slope response due to the bottomtrapped TRWs is important only in large $c$ value cases. In small $c$ value cases, we found the shelf/slope response is determined by surface-intensified wave components, whose existence is due to the planetary $\beta$ effect. For observed warm eddies with $c=3 \sim 5 \mathrm{~cm} \mathrm{~s}^{-1}$, the result of the present study suggests that both bottom-trapped TRWs and surface-intensified baroclinic waves are significant in determining the shelf/slope response. In cases when the localized forcing source oscillates in amplitude, we found that a localized response peak exists in the shelf/slope region. Along a fixed cross-slope section this response peak in the frictional case tends to shift offshore as the oscillating period of the forcing source increases. This result is opposed to the inviscid result, which shows that the response peak is independent of the forcing's oscillating period. The result for the frictional case is qualitatively consistent with that observed across the continental rise south of Cape Cod, thus suggesting the importance of bottom friction in determining the shelf/slope response.
\end{abstract}

\section{INTRODUCTION}

Many world shelf, slope, and rise regions are located near the western and northern edges of the interior ocean where boundary current meander and cutoff eddy activity is common and intense. Such activity can be an important energy source, causing current and temperature variations in neighboring ocean regions. Increasing evidence suggests that some of the low-frequency current fluctuations observed over shelf, slope, and rise regions (hereinafter abbreviated as shelf/slope regions) are indeed related to these offshore forcings. For example, on the basis of moored observations of current and temperature at 15 sites on the continental rise south of Cape Cod, Hogg [1981] found that a majority of fluctuation energy was contained in the frequency band spanning periods 8-108 days. By calculating the ray paths in a WKB model he concluded that the fluctuations of different wave periods have a common source near the Gulf Stream axis. Hogg's conclusion was further supported by synoptic field observations that revealed anomalous activity of the Gulf Stream during the first few months of the moorings [Luyten, 1977]. Shoreward propagating topographic waves were also detected by Louis et al. [1982] on the continental margin off Nova Scotia. Supported by sea surface temperature data of satellite imagery and other sources, Louis and Smith [1982] concluded that these wave bursts were associated with warm eddies shed by the Gulf Stream. Other examples of current fluctuations and their interaction with

\footnotetext{
${ }^{1}$ Now at Woods Hole Oceanographic Institution, Woods Hole, Massachusetts.

Copyright 1990 by the American Geophysical Union.

Paper number 89JC03685.

0148-0227/90/89JC-03685\$05.00
}

offshore forcings in the world shelf/slope regions can be found in the extensive review by Smith [1983].

Several studies have been proposed to investigate the shelf/slope response to offshore forcings. Under the assumption that the offshore forcing is a monochromatic wave which extends infinitely in the along-slope direction, Kroll and Niiler [1976] studied the onshore transmission of wave energy across a sloping bottom topography in a barotropic model. They found that high transmission can occur if the slope width is an integer number of the half cross-slope wavelength. This result for monochromatic wave forcings was also obtained by $O u$ and Beardsley [1980] in a stratified ocean model. In the stratified case, $\mathrm{Ou}$ and Beardsley further found that part of the incoming energy could scatter into baroclinic modes trapped to the steep slope. Recently, Chapman and Brink [1987] extended the study of monochromatic wave forcings by allowing the forcing to have a wide range of vertical structures, periods, and offshore locations. They found that the shelf/slope response depends largely upon the forcing period: when the period is shorter than 10 days, the response is dominated by near resonances of free coastally trapped waves; if the forcing period is long, the response is confined near the forcing region with a horizontal scale of the baroclinic Rossby radius.

When the offshore forcing is localized in space as in the real ocean, however, the shelf/slope responses can be considerably different from those caused by the monochromatic wave forcings. Kroll [1979] studied the shelf response in a barotropic ocean model by assuming that the forcing source is an isolated pressure cell with oscillating amplitudes. $\mathrm{He}$ found that such a forcing source can induce a distinct peak in the energy spectrum on the shelf. The barotropic radiation field of topographic Rossby waves generated by a localized source oscillator was also studied analytically by Louis and 
Smith [1982] and by Shaw and Peng [1987] using a numerical model. Both of these studies showed the importance of wave refraction and speculated that the wave refraction effect may explain the observed lack of reflected waves [e.g., Thompson, 1971]. By assuming that the localized forcing source propagates in the along-slope direction, Chapman and Brink [1987] investigated the shelf and slope circulations in a continuously stratified ocean model. They found that when it propagates close to the continental slope, such a forcing source induces a narrow jet moving in the direction against the topographic waves near the shelf break. Chapman and Brink [1987] attributed the existence of this narrow shelf break jet to arrested topographic waves [Csanady, 1978].

Although the shelf/slope response to the monochromatic wave forcing is relatively well understood, understanding of the shelf/slope response to localized offshore forcing is still insufficient. For cases where the forcing source has an oscillating amplitude, previous studies by Kroll [1979], Louis and Smith [1982], and Shaw and Peng [1987] have not considered how bottom friction and stratification may influence shelf/slope responses. In the present study we will show that the inclusion of bottom friction crucially influences the shelf/slope responses. For cases where the forcing source propagates in the along-slope direction, as considered by Chapman and Brink [1987], the role of the planetary $\beta$ effect has not yet been explored. Though small in comparison with the topographic $\beta$ effect in the lower layer slope region, the planetary $\beta$ effect is important because it induces surface-intensified wave motions and offshore barotropic wave motions. Both wave motions, as will be shown in the following investigations, are essential in transferring offshore wave energy onto the shelf/slope regions. The relative importance of these two wave motions in the shelf/slope response will be shown to depend upon the propagating speed of the forcing source.

In the present study these problems will be investigated in a two-layer $\beta$ plane ocean model. A two-layer model is adopted because we can more clearly interpret the numerical results when topographic changes in the slope region are large. Moreover, a two-layer model is a good simplification of the real ocean situation: observations by Thompson [1971] and Louis et al. [1982] show that current fluctuations at different depths below the thermocline are similar in phase and amplitude, although they may differ from those observed in the upper layer. It should, however, be pointed out that the density interface in the present layered model cannot intersect the bottom topography and the model's results are thus applicable only in the outer shelf and further offshore regions. Responses in the inner and middle shelf regions will not be pursued because low-frequency motions in these shelf regions are strongly affected by bottom friction and their responses are dynamically different from those in the outer shelf and slope regions.

\section{Model Formulation}

The model ocean shown in Figure 1 consists of an open ocean region, a relatively steep slope region, and a shallow outer shelf region. The right-hand coordinate system is fixed on the topography, wherein the $z$ axis is positive upward, the $y$ axis is parallel to the isobath, and the $x$ axis is directed positively offshore. In the model ocean the upper layer has a constant undisturbed depth $h$, and the lower layer has a

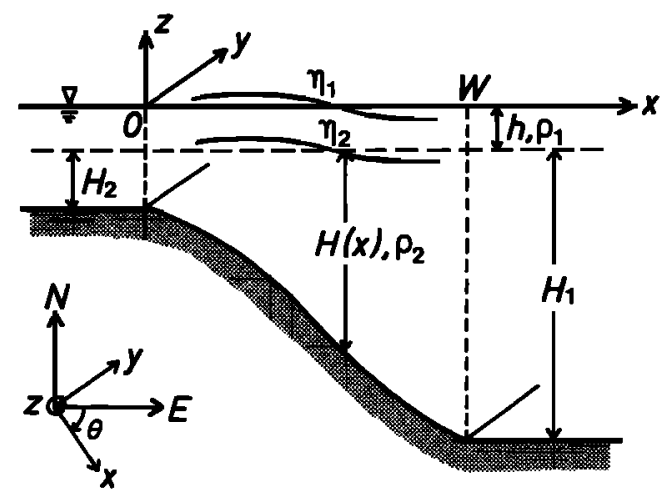

Fig. 1. Coordinate system and schematic diagram of the two-layer model ocean.

variable undisturbed depth $H(x)$ which is independent of $y$. The disturbed free surface is $\eta_{1}(x, y, t)$, and the interfacial displacement around its mean position is $\eta_{2}(x, y, t)$. The velocities are $\left(u_{j}, v_{j}\right)$, and the density is $\rho_{j}$, where $j=1$ and $j=2$ represent the variables in the upper layer and the lower layer, respectively.

First, we nondimensionalize the variables as follows:

$$
\begin{gathered}
(\bar{x}, \bar{y})=(x, y) / L \quad(\bar{h}, \tilde{H})=(h, H) / H_{1} \quad \tilde{t}=t f_{0} \\
\tilde{f}=f / f_{0} \quad\left(\tilde{u}_{j}, \tilde{\nu}_{j}\right)=\left(u_{j}, v_{j}\right) / U \\
\bar{\eta}_{1}=\eta_{1} /\left(f_{0} L U / g\right) \quad \bar{\eta}_{2}=\eta_{2} /\left(f_{0} L U / g^{\prime}\right)
\end{gathered}
$$

where $L$ is a horizontal scale of the slope width, $U$ is a characteristic horizontal velocity which is taken to be the maximum velocity of the offshore forcing eddy, $H_{1}$ is the lower layer thickness at the open ocean, $f_{0}$ is the Coriolis parameter at the reference latitude, and $g^{\prime}=\left(\rho_{2}-\rho_{1}\right) g / \rho_{2}$ is the reduced gravity constant. Under the hydrostatic approximation the linearized momentum equations and the depth-averaged continuity equations become (after dropping the tildes for the nondimensional variables)

$$
\begin{gathered}
u_{1 t}-f v_{1}=-\eta_{1 x} \\
v_{1 t}+f u_{1}=-\eta_{1 y} \\
u_{1 x}+v_{1 y}-\eta_{2 t} / S h+g^{\prime} \eta_{1 t} / g S h=0 \\
u_{2 t}-f v_{2}=-\eta_{1 x}-\eta_{2 x} \\
v_{2 t}+f u_{2}=-\eta_{1 y}-\eta_{2 y} \\
u_{2 x}+v_{2 y}+u_{2} H_{x} / H+\eta_{2 t} / S H=\frac{1}{2} E_{v}^{1 / 2}\left(v_{2 x}-u_{2 y}\right)
\end{gathered}
$$

In (2) and (3), subscripts $(x, y, t)$ denote partial differentiation, and $S=g^{\prime} H_{1} / f_{0}^{2} L^{2}$ is the stratification parameter. The Coriolis parameter $f$ is $1+\alpha x+\beta y$, where $(\alpha, \beta)=(-\sin \theta$, $\cos \theta) \beta_{0} \mathrm{~L} / \mathrm{f}_{0}, \theta$ is the angle measured clockwise from the east to the $x$ axis, and $\beta_{0}$ is the dimensional northward gradient of $f$. In $(3 c)$ the term on the right-hand side represents Ekman pumping from the bottom boundary layer, where $E_{v}=2 A_{v}$ l $f_{0} H^{2}$ is the vertical Ekman number and $A_{v}$ is the vertical eddy viscosity [e.g., Pedlosky, 1979].

In the following analysis we will assume $E_{v}$, the depth ratio of the bottom Ekman layer to the lower layer, to be small and independent of $x$. We will also omit the surface elevation term $g^{\prime} \eta_{1 t} / g S h$ in (2c) because it is negligibly small 
in comparison with the interfacial elevation term $-\eta_{2 t} / S h$. Eliminating $u_{j}$ and $v_{j}$ from (2) and (3) and neglecting $\partial^{2} / \partial t^{2}$ against $f_{0}^{2}$ (since we are interested in responses with low frequencies), we obtain the following two equations governing $\eta_{1}$ and $\eta_{2}$ :

$$
\begin{array}{r}
\left(\eta_{1 x x}+\eta_{1 y y}+\eta_{2} / S h\right)_{t}+\beta \eta_{1 x}-\alpha \eta_{1 y}=0 \\
\left(\eta_{2 x x}^{\prime}+\eta_{2 y y}^{\prime}+\eta_{2 x}^{\prime} H_{x} / H-\eta_{2} / S H\right)_{t}+\beta \eta_{2 x}^{\prime} \\
-\alpha \eta_{2 y}^{\prime}-\eta_{2 y}^{\prime} H_{x} / H=\frac{1}{2} E_{v}^{1 / 2}\left(\eta_{2 x x}^{\prime}+\eta_{2 y y}^{\prime}\right)
\end{array}
$$

where $\eta_{2}^{\prime} \equiv \eta_{1}+\eta_{2}$. In deriving (4) and (5) we have also used the assumptions $|\beta| \ll|\partial / \partial y|$ and $|\alpha| \ll|\partial / \partial x|$, which are consistent with the $\beta$ plane approximation.

We will divide the investigation into two cases according to the pattern of the forcing source movement at the offshore boundary. When the forcing source propagates in the alongslope direction or has an oscillating amplitude, the offshore forcing functions will be assumed to be

$$
\left(\eta_{1}, \eta_{2}^{\prime}\right)\left(x_{0}, y, t\right)=(1, \gamma) A_{0} \exp \left[-(y-c t)^{2} / d^{2}\right]
$$

or

$$
\left(\eta_{1}, \eta_{2}^{\prime}\right)\left(x_{0}, y, t\right)=(1, \gamma) A_{0} \exp \left[-y^{2} / d^{2}-i \sigma_{f} t\right]
$$

respectively. The Gaussian-shaped forcing source of (6) was also used in the study by Chapman and Brink [1987]. In (6) and (7), $x_{0}$ denotes the offshore distance where the forcing is imposed, $\gamma$ denotes the forcing strength of the lower layer relative to that of the upper layer, and $A_{0}, c, \sigma_{f}$, and $d$ denote the amplitude, the along-slope propagating speed, the oscillating frequency, and the half width of the forcing source, respectively.

Shelf/slope responses to these forcing sources are obtained by first Fourier decomposing the forcing functions

$$
\begin{array}{r}
\left(Z_{1}, Z_{2}^{\prime}\right)\left(x_{0}, m, \sigma\right)=\int_{-\infty}^{\infty} \int_{-\infty}^{\infty}\left(\eta_{1}, \eta_{2}^{\prime}\right)\left(x_{0}, y, t\right) \\
\cdot \exp [-i(m y-\sigma t) d y d t
\end{array}
$$

To each forcing component in (8) the shelf/slope response $\left(Z_{1}, Z_{2}^{\prime}\right)(x, m, \sigma)$ is then calculated from

$$
\begin{aligned}
Z_{1 x x}+(i \beta / \sigma) Z_{1 x}+ & \left(-m^{2}+\alpha m / \sigma-1 / S h\right) Z_{1}+Z_{2}^{\prime} / S h=0 \\
\varepsilon Z_{2 x x}^{\prime}+(i \beta / \sigma+ & \left.H_{x} / H\right) Z_{2 x}^{\prime}+\left(-\varepsilon m^{2}+\alpha m / \sigma\right. \\
& \left.-1 / S H-m H_{x} / H \sigma\right) Z_{2}^{\prime}+Z_{1} / S H=0
\end{aligned}
$$

where $\varepsilon=1+E_{v}^{1 / 2} i / 2 \sigma$. Finally, the total response in the shelf/slope region is obtained by summing up all the Fourier component responses:

$$
\begin{array}{r}
\left(\eta_{1}, \eta_{2}^{\prime}\right)(x, y, t)=\frac{1}{4 \pi^{2}} \int_{-\infty}^{\infty} \int_{-\infty}^{\infty}\left(Z_{1}, Z_{2}^{\prime}\right)(x, m, \sigma) \\
\cdot \exp [i(m y-\sigma t)] d \sigma d m
\end{array}
$$

If the slope has large depth changes, the conventional "weak slope assumption" (in which $H(x)$ is assumed to be constant except when differentiated [e.g., Allen, 1984]) cannot be used to solve (9) and (10) [Gratton and LeBlond, 1986]. Most of the world continental slope regions, however, have large depth changes. In the present study we allow for bottom depth changes with arbitrary amplitudes by numerically solving (9) and (10). The boundary conditions for the model are chosen such that (1) the waves reflected from the slope propagate freely through the offshore open boundary and (2) over the shelf region we consider only transmitted waves. Waves reflected from the shore are not considered because dissipation at the shallow shelf region effectively damps out the transmitted wave energies [Kroll and Niiler, 1976; Chapman and Brink, 1987].

The numerical technique used in solving (9) and (10) is as follows: We first divide the depth-varying region into $N+1$ subregions. Assuming the depth in each subregion to be constant (the mean value of the subregion), we can write solutions for each subregion as a sum of transmitted and reflected barotropic and baroclinic wave components:

$$
\begin{aligned}
& Z_{1}=\sum_{n=1}^{4} A_{n} \exp \left(i k_{n} x\right) \\
& Z_{2}^{\prime}=\sum_{n=1}^{4} \alpha_{n} A_{n} \exp \left(i k_{n} x\right)
\end{aligned}
$$

In (12) and (13), wave numbers $k_{n}$ are solutions of the following biquadratic equation:

$$
\begin{aligned}
k^{4}+\left(a_{1}+a_{2}\right) k^{3}+\left(a_{1} a_{2}+b_{1}+b_{2}\right) k^{2} & \\
+\left(a_{1} b_{2}+a_{2} b_{1}\right) k+\left(b_{1} b_{2}-c_{1} c_{2}\right) & =0
\end{aligned}
$$

where

$$
\begin{array}{ccc}
a_{1}=\beta / \sigma \quad a_{2}=\beta / \sigma \varepsilon & c_{1}=1 / S h \quad c_{2}=1 / S H \varepsilon \\
b_{1}=m^{2}-\alpha m / \sigma+c_{1} & b_{2}=m^{2}-\alpha m / \sigma \varepsilon+c_{2}
\end{array}
$$

and the separation constants $\alpha_{n}$ satisfy $\alpha_{n}=\left(k_{n}^{2}+a_{1} k_{n}+\right.$ $\left.b_{1}\right) / c_{1}$.

Across the depth discontinuity, conditions

$$
\begin{aligned}
& {\left[Z_{1}\right]_{-}^{+}=0 \quad\left[Z_{2}^{\prime}\right]_{-}^{+}=0} \\
& {\left[Z_{1 x}\right]_{-}^{+}=0 \quad\left[H\left(\sigma \varepsilon Z_{2 x}^{\prime}-m Z_{2}^{\prime}+i \beta Z_{2}^{\prime} / 2\right)\right]_{-}^{+}=0}
\end{aligned}
$$

are required to ensure the continuity of mass flux and pressure, where [ $]_{-}^{+}$denotes the difference of the bracketed quantities across the discontinuity [e.g., LeBlond and Mysak, 1978]. By applying these matching conditions at $N$ points of depth discontinuity and by using the above mentioned boundary conditions, we can obtain a set of $4 N$ linear equations in terms of $4 N$ unknown wave amplitudes. The response $\left(Z_{1}, Z_{2}^{\prime}\right)(x, m, \sigma)$ is calculated by solving this set of linear equations. In the actual numerical calculations, $N$ is chosen to be 31 , which we found gives fine enough resolution for the present problem.

To simplify the analysis of the numerical results, we will concentrate our discussion on a simple basin case in which the lower layer has a depth configuration of

$$
\begin{array}{lr}
H(x)=H_{2} & \multicolumn{1}{c}{x \leq 0} \\
H(x)=H_{2} \exp (M x) & 0<x<W \\
H(x)=1 & W \leq x
\end{array}
$$

where $\mathrm{H}_{2}$ and $\mathrm{M}\left(\equiv \mathrm{H}_{x} / H\right)$ are both constant. Despite its simplicity this basin case provides us with the essential 
characteristics of the shelf/slope response in a stratified ocean of varying depth. In the following analysis we further assume that the offshore forcing source is barotropic (that is, $\gamma=1$ ) and fix the external parameters for the figures at $H_{2}=$ $h=0.143, M=1.946, W=1.0, S=0.411, E_{v}^{1 / 2}=0.0025,(\alpha$, $\beta)=(0.0,0.0488), x_{0}=2.0$, and $d=0.4$. In the formulation and discussion, however, these parameters will be as general as possible.

\section{Shelf/Slope Responses to Along-Slope Propagating Forcing Sources}

First, we will investigate the shelf/slope response caused by the propagating forcing source (6) in the inviscid limit. Fourier decomposing (6) yields

$$
\begin{aligned}
\left(Z_{1}, Z_{2}^{\prime}\right) & \left(x_{0}, m, \sigma\right) \\
= & (1, \gamma) A_{0} 2 d \pi^{3 / 2} \exp \left(-d^{2} m^{2} / 4\right) \delta(\sigma-m c)
\end{aligned}
$$

where $\delta$ is the Dirac delta function. The appearance of $\delta(\sigma-$ $m c$ ) in (17) implies that given a propagating speed $c$, only wave components that satisfy $\sigma=m c$ contribute to the shelf/slope response. Since cutoff warm eddies generally propagate with the slope to the right [Joyce, 1984], we will concentrate our investigation on the case of $c<0$.

In Figures $2 a$ and $2 b$ we show the $\left(\eta_{1}, \eta_{2}^{\prime}\right)$ patterns and the along-slope distributions of the energy density averaged over the shelf when $c$ is -0.0055 and -0.0065 , respectively. These $c$ values correspond to the dimensional speeds of -5.5 $\mathrm{cm} \mathrm{s}^{-1}$ and $-6.5 \mathrm{~cm} \mathrm{~s}^{-1}$ when $f_{0}=10^{-4} \mathrm{~s}^{-1}$ and $L=100$ $\mathrm{km}$. Notice that the velocities in the upper and lower layers are nearly proportional to the gradient of the $\eta_{1}$ and $\eta_{2}^{\prime}$ contours. The shelf/slope responses in the two cases are considerably different, even though the propagating speed differs by only 0.001: In Figure $2 a$, strong responses are induced over both the shelf and lower layer slope region shoreward of the forcing source, but in Figure $2 b$ the induced responses in the shelf/slope regions are weak, and we find instead large-amplitude reflected waves in the offshore ocean region. The difference in response between the two cases is also clear from the shelf energy density distributions shown in Figure 2; the maximum energy density value in the case $c$ $=-0.0055$ is 4 times larger than in the case $c=-0.0065$.

The shelf/slope response's dependence upon the propagating speed can be understood as follows. Studies by Kroll and Niiler [1976] and Ou and Beardsley [1980] have shown that when a monochromatic wave forcing is imposed in the offshore ocean, the energy transmission onto the shelf region depends largely on intrinsic phase changes of the incident wave across the slope. Maximum energy transmission occurs when the intrinsic phase change is $n \pi$, while the transmission is minimum when it is $(n+1 / 2) \pi$, where $n$ is an integer (the Ramschauer effect [Rhines, 1969]). In the present two-layer model, this dynamics also holds true. The intrinsic phase change of bottom-trapped topographic Rossby waves (TRWs) across the slope in the two-layer model can be approximated by

$$
I=K_{s} W-\left(e^{M W}-1\right) / M S K_{s}
$$

(see (A4)), where $K_{s}$ satisfies the dispersion relation of nondivergent barotropic Rossby waves:

$$
K_{s}^{2}=\beta^{2} / 4 \sigma^{2}+(\alpha-M) m / \sigma-m^{2}-M^{2} / 4
$$

In Figure 3 the $(m, 1 / \sigma)$ dependence of $I=n \pi$ is shown by dashed lines. Also shown in Figure 3 are $T_{\text {tro }}$ and $T_{c l i}$, the energy flux transmission coefficients (FTCs) transmitted from the offshore barotropic wave to the shelf barotropic and baroclinic waves. As in the work by Kroll and Niiler [1976], the FTCs are defined as the ratio of the $x$ component energy flux between the incident and transmitted waves. As can be anticipated from earlier studies, maximum FTCs appear in parameter ranges around $I=n \pi$ (a more detailed discussion is given in the appendix).

In the case of propagating localized forcing sources it is important to notice that the $K_{s}^{2}$ value in (19) is essentially determined by $-m M / \sigma$ because the vortex stretching term predominates in the vorticity balance over the slope region. The fact that $K_{s}^{2} \sim(-m M / \sigma)$ means that for a given $c=\sigma / m$, all contributing wave components induce bottom-trapped TRWs with the same $K_{\mathrm{s}}$ and hence $I$ values. From the results of Figure 3 we can thus expect maximum (minimum) shelf/ slope responses to occur if the given propagating speed results in $I=\mu \pi$ with $\mu$ being an integer (an integer plus $1 / 2$ ). Using (18) and (19) to relate $\mu$ with $c$,

$$
\mu \sim \frac{1}{\pi}\left(-\frac{M}{c}\right)^{1 / 2}\left[W+\frac{c}{M^{2} S}\left(e^{M W}-1\right)\right]
$$

we found that for $c=-0.0055$ and -0.0065 the corresponding $\mu$ value is 6 and 5.5, respectively. This difference in $\mu$ explains why the shelf/slope response differed significantly between Figures $2 a$ and $2 b$, even though the difference in the propagating speed itself was small.

The $\mu$ dependence of shelf/slope responses becomes even clearer in Figure 4, wherein we have plotted the maximum energy density values induced on the shelf against the $c$ values. Note that the large solid circles in the figure denote cases where $\mu$ are integers, while the open circles denote cases where $\mu$ are integers plus $1 / 2$. From Figure 4 it is also clear that the energy density maximum value increases as $|c|$ decreases. This is due to the increase in the number of incident Rossby wave components permissible in the offshore ocean as $|c|$ decreases

Next, we examine how bottom friction, which always influences low-frequency wave motions, modifies the above results obtained in the inviscid limit. In Figure 5 we show the shelf/slope response under the same forcing situation as in Figure $2 a$ except for $E_{v}^{1 / 2}=0.0025$. In the figure the energy density distribution is evaluated at the shelf break, and velocity vectors are superimposed upon the $\left(\eta_{1}, \eta_{2}^{\prime}\right)$ contours. Notice that an $E_{v}^{1 / 2}$ value of 0.0025 in the present case corresponds to a bottom Ekman layer of $5 \mathrm{~m}$ [cf. Ou and Beardsley, 1980]. Comparing the $\eta_{2}^{\prime}$ contours in Figure 5 with those in Figure $2 a$, we find that wave motions in the lower layer slope region are greatly reduced. This large reduction occurs because most bottom-trapped TRWs induced in the case of $c=-0.0055$ have small decay scales of the same order as the slope width, i.e., $k_{i s}^{-1} \sim 1.0$ (see Figure 6 , where $k_{i s}^{-1}$ is the $e$-folding decay scale of the bottomtrapped TRWs in the cross-slope direction estimated from (A1)). In the upper layer of the slope region, however, we find that the wave motion has practically the same amplitude as in the inviscid case. The upper layer wave motion is not influenced by bottom friction because surface-intensified wave components in the slope region generally have decay 
(a)
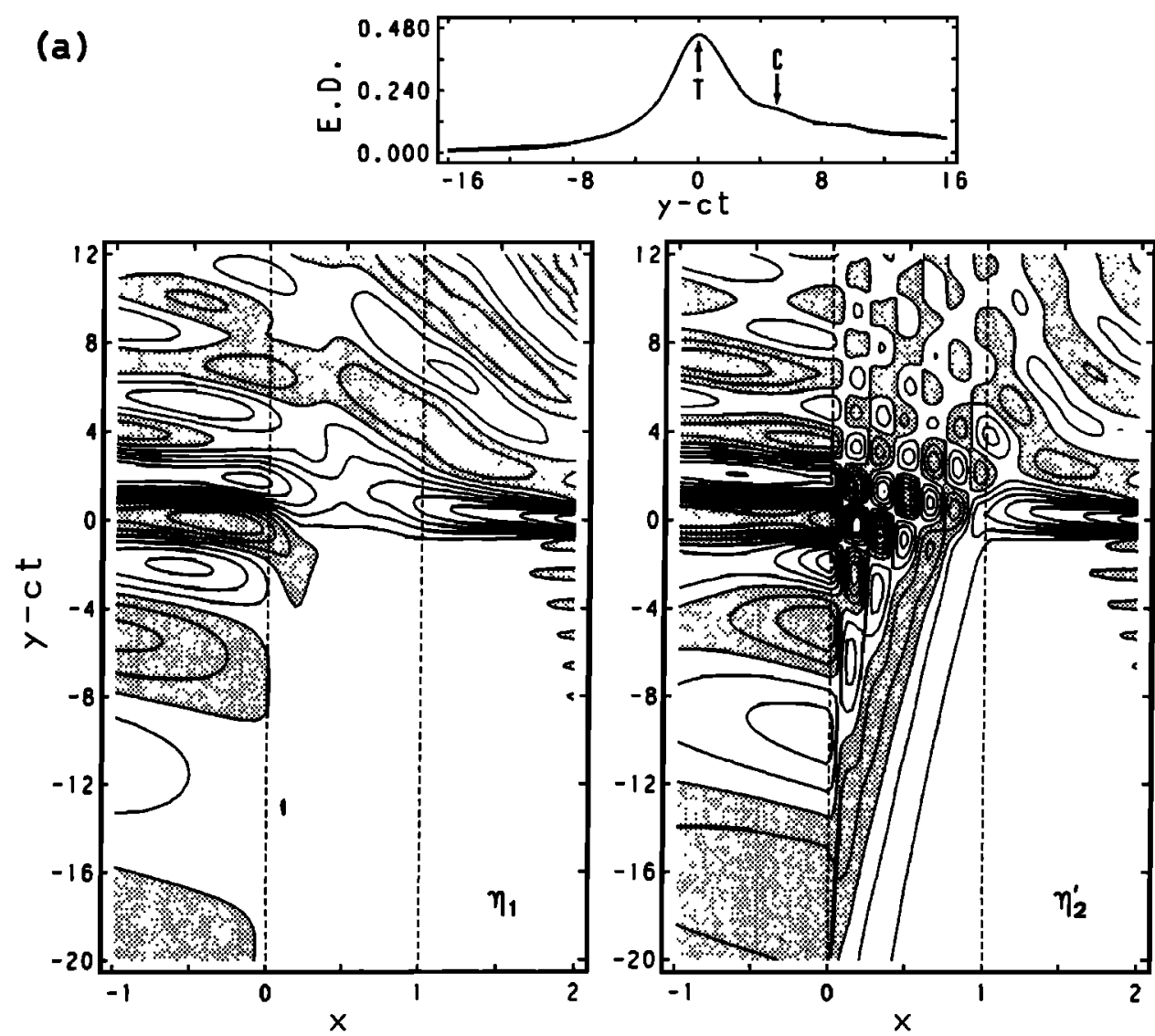

(b)
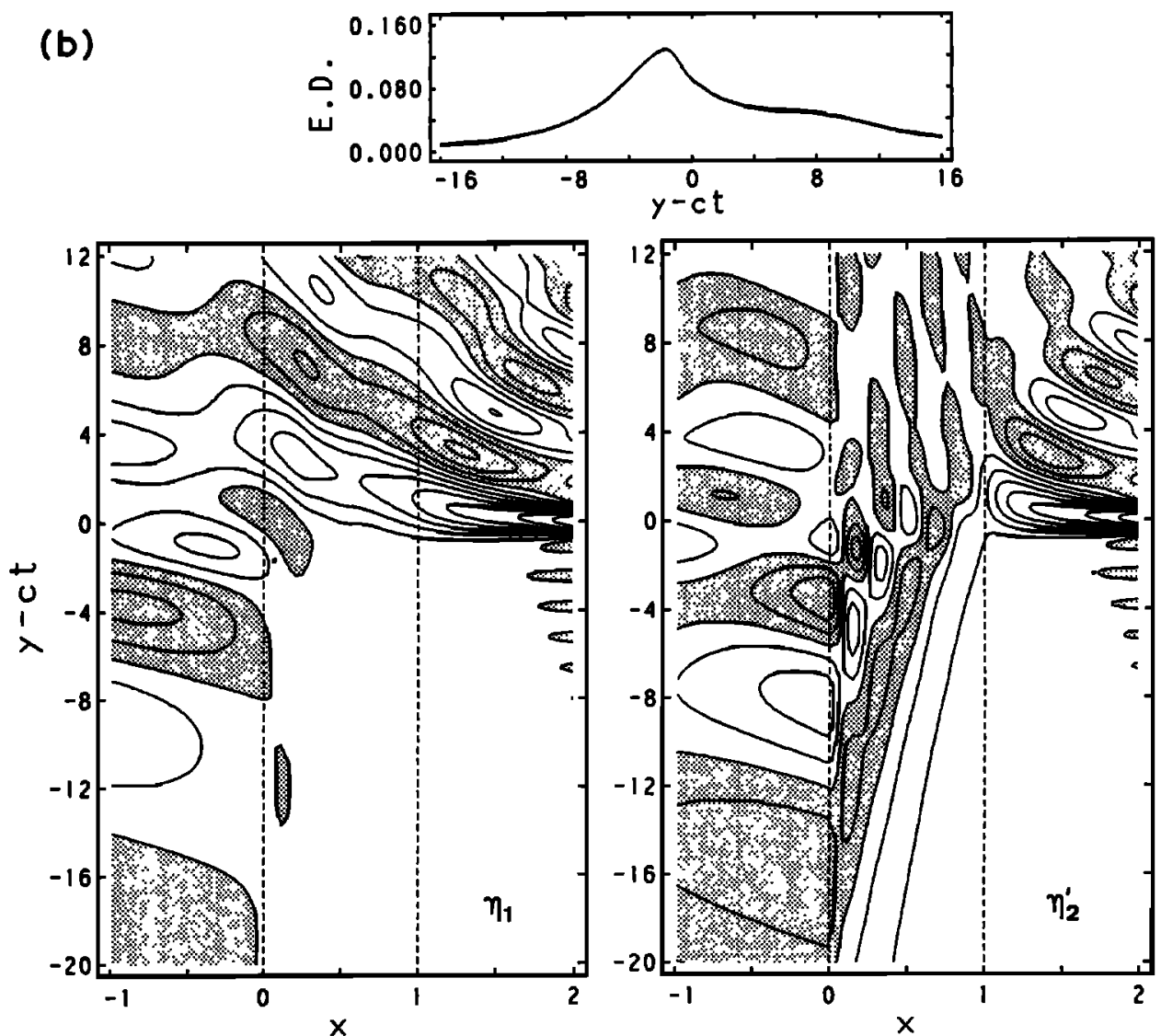

Fig. 2. The $\left(\eta_{1}, \eta_{2}^{\prime}\right)$ patterns and averaged shelf energy density distributions induced by localized forcing sources propagating in the along-slope direction when $E_{v}^{1 / 2} \rightarrow 0$. The speed of propagation is $(a)-0.0055$ and $(b)-0.0065$. The contour interval values for $\eta_{1}$ and $\eta_{2}^{\prime}$ are $0.1 A_{0}$, and the unit for the energy density distribution is $A_{0}^{2}$, where $A_{0}$ is the amplitude of the forcing source. 
(a) Ttro

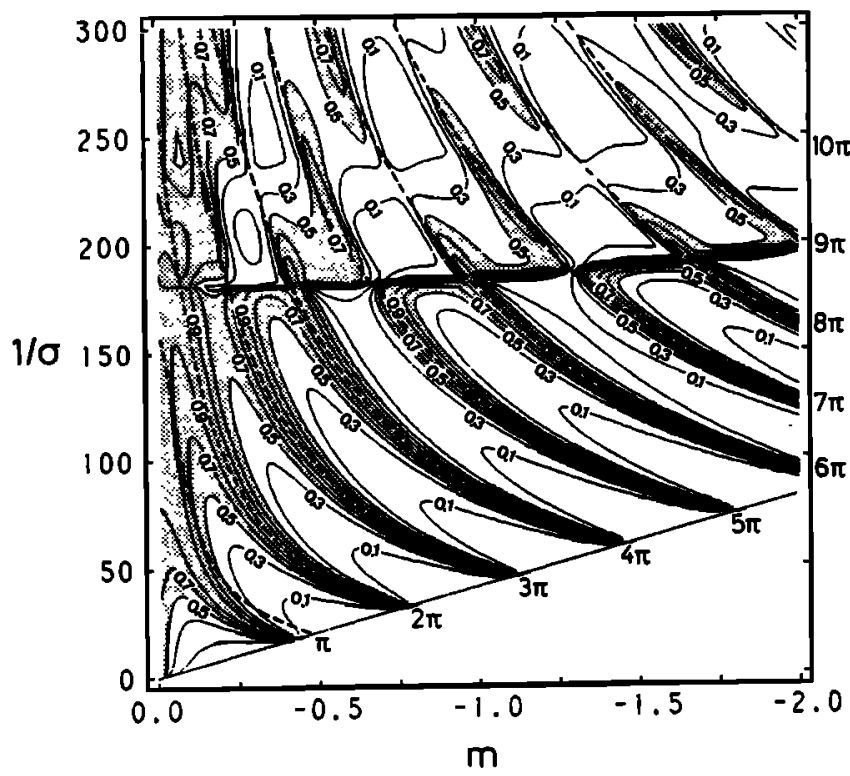

(b) $\mathrm{T}$ cli

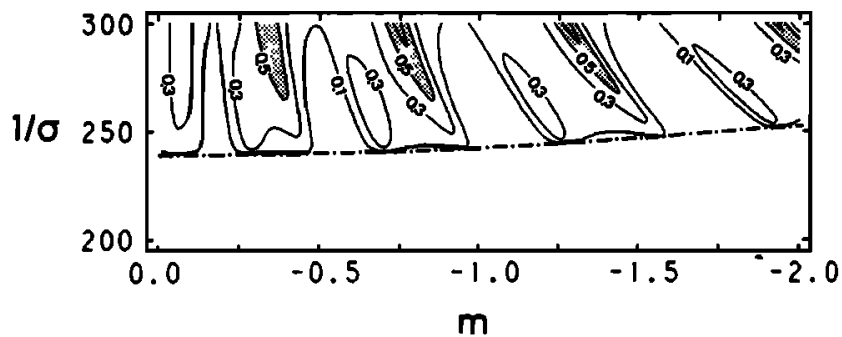

Fig. 3. Energy flux transmission coefficients of (a) $T_{\text {tro }}$ and (b) $T_{\text {cli }}$ across a continental slope in a two-layer model. The mode of the incident wave is barotropic. The stippled areas indicate highly transmissible ranges with coefficients exceeding 0.5 . The dashed lines indicate isopleths of $I$, which defines the phase change of the bottom-trapped TRWs across the slope region.

scales at least 1 order larger than the slope width (not shown).

The tendency for bottom friction to primarily damp out bottom-trapped TRWs in the slope region also has a significant effect on the outer shelf response. Comparing the shelf energy density distribution in Figure 5 with that in Figure $2 a$ reveals that the energy peak induced by the bottom-trapped TRWs is greatly reduced in the frictional case (arrow T); as a result, the energy peak induced by the surface-intensified waves (arrow C) is relatively dominant in the shelf energy density field. Notice that a maximum energy density value of $0.1 a_{0}^{2}$ in Figure 5 indicates that the maximum velocity over the outer shelf is of the order of $0.2 v_{m}$, where $v_{m} \equiv$ $2^{1 / 2} A_{0} d^{-1} e^{-1 / 2}$ is the maximum velocity of the offshore forcing eddy.

Since the decay scales of the bottom-trapped TRW components become even smaller as the propagating speed of the forcing source decreases (see Figure 6), we expect the shelf energy peak induced by the bottom-trapped TRWs to decline further. In Figure 7 we have plotted the maximum energy density values obtained in the frictional cases with different $c$ values: Circles denote energy peaks induced by the bot-

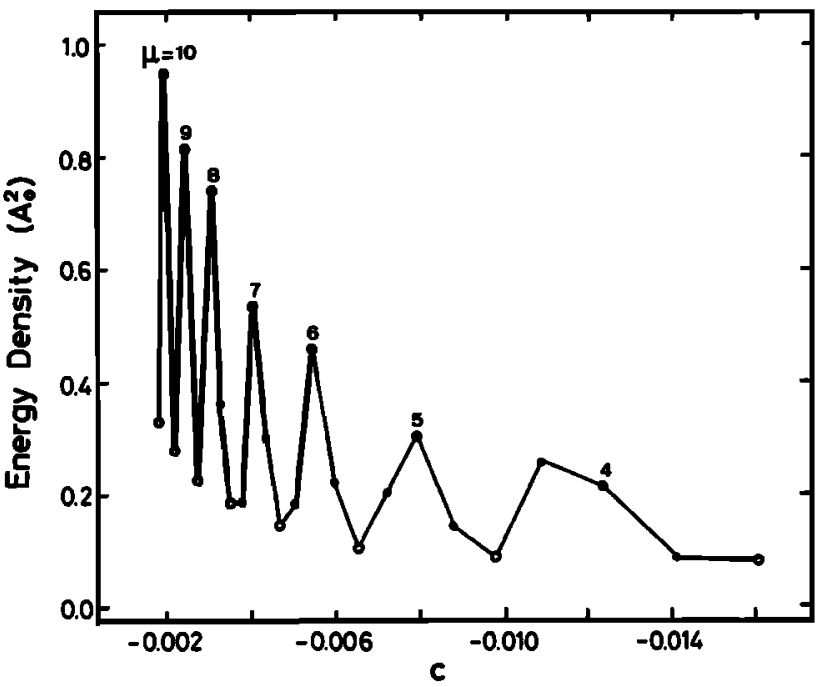

Fig. 4. Maximum energy density values on the shelf induced by forcing sources with different propagating speeds $\left(E_{v}^{1 / 2} \rightarrow 0\right)$. In the figure, large solid circles denote cases in which the corresponding $\mu$ values are integers, while open circles denote cases in which the $\mu$ values are integers plus $1 / 2$.

tom-trapped TRWs, and squares denote those induced by the surface-intensified waves. As expected, the energy peak induced by the bottom-trapped TRWs decrease as the propagation of the forcing source slows down. Since the intrinsic phase changes of the bottom-trapped TRWs are essentially not influenced by the inclusion of bottom friction (see (A3)), the $\mu$ dependence, though much less distinctive than in Figure 4, is still discernable in the solid line of Figure 7. When the propagating speed of the forcing source is small, we find that the energy peaks induced by the surfaceintensified waves surpass those induced by the bottomtrapped TRWs. The tendency for peak values induced by the surface-intensified waves to increase in slower-propagating forcing cases is due to the increase in the number of permissible surface-intensified wave components as $|c|$ decreases.

\section{Shelf/Slope Responses to Forcing Sources With Oscillating Amplitudes}

In this section we consider the shelf/slope response caused by a localized forcing source which oscillates in amplitude. As the wave frequency in this case is fixed at $\sigma_{f}$, we need to decompose the forcing function (7) only in terms of the along-slope wave number:

$$
\begin{aligned}
& \left(Z_{1}, Z_{2}^{\prime}\right)\left(x_{0}, m, \sigma_{f}\right) \\
& \quad=(1, \gamma) A_{0} d \pi^{1 / 2} \exp \left[-d^{2} m^{2} / 4-i \sigma_{f} t\right]
\end{aligned}
$$

Two remarkable differences exist between this case and the propagating forcing case in section 3 . First, given a propagating speed $c$ in the case of section 3 , all wave components tend to have a similar FTC value and decay scale. In the present case, however, transmission coefficients and decay scales are different for each individual forcing component (see Figures 3 and 6). Second, the parameters $\sigma$ and $m$ in the last section are locked by $\sigma=m c$, thus limiting dispersion to the cross-slope direction. In the present oscillating case, 

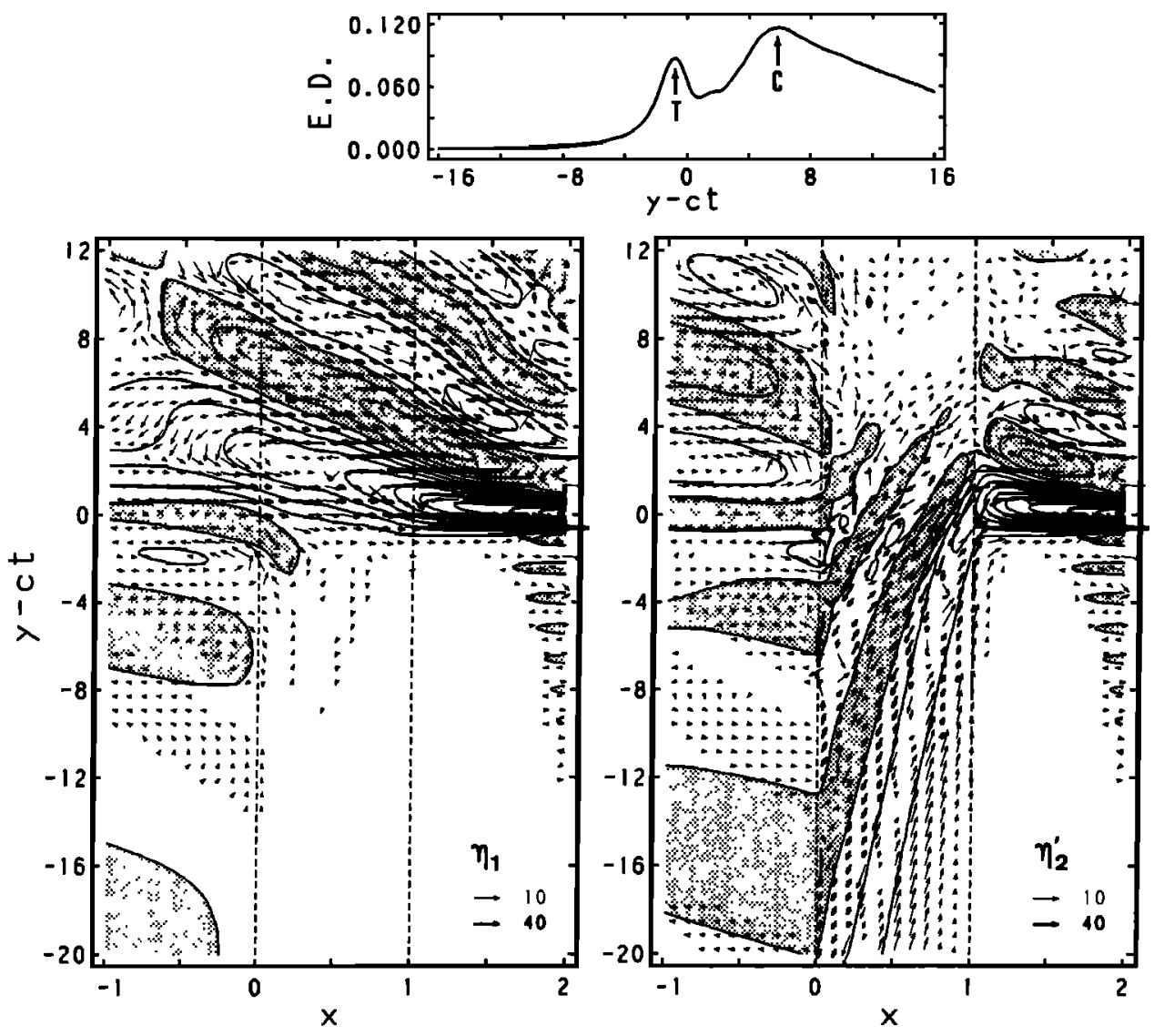

Fig. 5. As in Figure $2 a$ except for the inclusion of bottom friction $\left(E_{v}^{1 / 2}=0.0025\right)$. The contour interval values for $\eta_{1}$ and $\eta_{2}^{\prime}$ are $0.075 A_{0}$, and the unit for velocity vectors is $0.03 A_{0}$. In the along-slope energy density distribution, arrows $\mathrm{T}$ and $\mathrm{C}$ indicate the energy density maxima induced by the bottom-trapped TRWs and the surface-intensified baroclinic waves, respectively.

dispersion occurs in both the cross-slope and along-slope directions over the slope region.

In Figure 8 we show the $\left(\eta_{1}, \eta_{2}^{\prime}\right)$ pattern and the shelf energy density distribution in the inviscid limit when $1 / \sigma_{f}=$ 110. For $f_{0}=10^{-4} \mathrm{~s}^{-1}$, this $1 / \sigma_{f}$ value corresponds to a forcing oscillating period of 80 days. After barotropic Rossby waves induced by the forcing source in the offshore ocean reach the slope region, we find that wave motions in the upper layer diminish shoreward gradually because no surface-intensified waves are excited at this $1 / \sigma_{f}$ value. In the lower layer slope region, on the other hand, bottomtrapped TRWs are induced and dispersed in the $-y$ direction. The energy density produced by these bottom-trapped TRWs is shown in Figure 8 to have a distinct peak at $y=$ -5.4 over the shelf. Notice that wave motions will reappear in the upper layer after the bottom-trapped TRWs reach the shelf break. This is because coupling between the upper and lower layer motions strengthens in flat ocean regions.

When bottom friction is included, as shown in Figure 9, we find that the $\left(\eta_{1}, \eta_{2}^{\prime}\right)$ pattern is basically similar to that obtained in the above inviscid case. A noteworthy difference between the two cases, however, is that the shelf energy density peak appears at $y=-8.1$ in the frictional case but at $y=-5.4$ in the inviscid case. Moreover, numerical calculations with various oscillating frequency values reveal that while the location of the energy density peak in the inviscid case has a relatively weak dependence on the $1 / \sigma_{f}$ values (solid circles in Figure 10), the energy density peak in the frictional cases tends to shift to a farther $-y$ location as the oscillating period of the forcing source becomes long (open circles in Figure 10).

In order to explain this difference in $1 / \sigma_{f}$ dependence between the frictional and inviscid cases as well as clarify the parameters determining the location of the energy density peak, we will simplify our discussion below to a barotropic ocean model. We can do so because coupling between the layer motions in the slope region is negligibly weak [Suginohara, 1981], rendering the dynamics determining the energy density peak induced by the bottom-trapped TRWs the same in both the barotropic and baroclinic cases.

Following Kroll and Niiler [1976], we can express the shelf response to a monochromatic wave forcing in the present model case with bottom friction by

$$
\begin{array}{r}
Z\left(x, m, \sigma_{f}\right)=G(m) Z\left(x_{0}, m, \sigma_{f}\right) \exp (-M W / 2 \varepsilon) \\
\cdot \exp \left[i\left(\bar{K}-\bar{K}_{s}\right) W+i\left(\bar{K}-\beta / 2 \sigma_{f} \varepsilon\right)\left(x-x_{0}\right)\right]
\end{array}
$$

where

$$
\begin{gathered}
\bar{K}^{2}=\beta^{2} / 4 \varepsilon^{2} \sigma_{f}^{2}-m^{2}+m \alpha / \varepsilon \sigma_{f} \\
\bar{K}_{s}^{2}=\beta^{2} / 4 \varepsilon^{2} \sigma_{f}^{2}-m^{2}+m(\alpha-M) / \varepsilon \sigma_{f}-M^{2} / 4 \varepsilon^{2} \\
G(m)=4 \bar{K}_{s} /\left[2 \bar{K} \bar{K}_{s}\left(1+e^{-i 2 \bar{K}_{s} W}\right)\right. \\
\left.+\left(\bar{K}^{2}+\bar{K}_{s}^{2}+M^{2} / 4 \varepsilon^{2}\right)\left(1-e^{-i 2 \bar{K}_{s} W}\right)\right]
\end{gathered}
$$

In (22) the offshore forcing function $Z\left(x_{0}, m, \sigma_{f}\right)$ is equal to $Z_{1}\left(x_{0}, m, \sigma_{f}\right)$ in (21). By inverting the Fourier transform and 


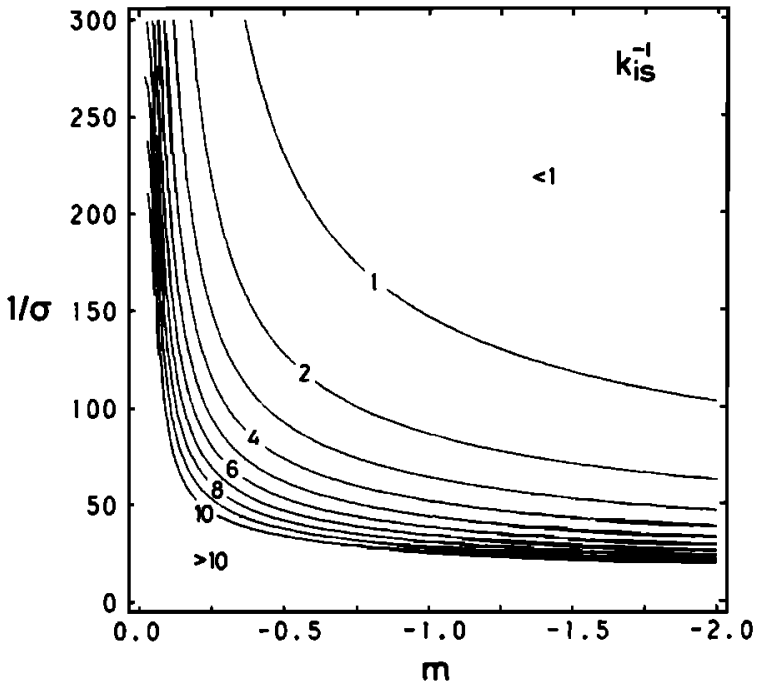

Fig. 6. $E$-folding decay scales of the bottom-trapped TRWs in the cross-slope direction, where the vertical Ekman number $\left(E_{v}^{1 / 2}\right)$ is 0.0025 . The dimensional unit for the decay scale is the slope width.

assuming $\Delta=E_{v}^{1 / 2} / 2 \sigma \ll 1$ (notice that $\varepsilon \equiv 1+\Delta$ ) we can write the shelf response, to $O\left(\Delta^{2}\right)$, as follows:

$$
\eta(x, y, t)=Q(t) \int_{-\infty}^{\infty} F(m) e^{i \phi(m)} d m
$$

where

$$
\begin{gathered}
Q(t)=\frac{1}{2(\pi)^{1 / 2}} d A_{0} \exp \left[\frac{M W}{2 \varepsilon} i \sigma_{f} t-\frac{i \beta\left(x-x_{0}\right)}{2 \sigma}\right] \\
\phi(m)=K_{r o}\left(W+x-x_{0}\right)+m y-K_{s} W
\end{gathered}
$$

$F(m)=G(m) \exp \left[-m^{2} d^{2} / 4+\left(K_{i s}+\beta \Delta / 2 \sigma\right) W\right.$

$$
\left.-\left(K_{i o}+\beta \Delta / 2 \sigma\right)\left(W+x-x_{0}\right)\right]
$$

$\operatorname{In} \phi(m)$ and $F(m), K_{r o}=\operatorname{Re}(\bar{K}), K_{i o}=\operatorname{Im}(\bar{K})$, and $K_{i s}=\operatorname{Im}$ $\left(\bar{K}_{s}\right)$, respectively. Since the magnitude of the $u$ component velocity over the shelf is much larger than the $v$ component in the present case (Figure 9), we can evaluate the energy density distribution over the shelf region by

$\bar{E}(x, y) \sim \frac{1}{2}\left|-\eta_{y}\right|^{2}=\frac{1}{2}\left|Q(t) \int_{-\infty}^{\infty} m F(m) e^{i \phi(m)} d m\right|^{2}$

By setting $\partial \phi(m) / \partial m=0$ we find that the stationary phase of the integral in (24) is given by

$y+\frac{\partial K_{r o}}{\partial m}\left(W+x-x_{0}\right)+\left[\frac{m+\left(M-\alpha / 2 \sigma_{f}\right)}{K_{s}}\right] W=0$

Physically, this equation determines the ray path that is followed by the wave component with wave number $m$ and frequency $\sigma_{f}$ [e.g., Kroll, 1979]. Notice that the third term in (25), which expresses the $y$ deflection by the TRWs in the slope region, greatly exceeds the second term, expressing the $y$ deflection by the Rossby waves in the offshore and shelf regions. In Figure 11 we depict the $(m, 1 / \sigma)$ dependence of $\delta=\tan ^{-1}(-W / y)$, the angle between the ray path and the $-y$ axis in the slope region. It is clear from the figure that given a fixed oscillating period a wave component with a smaller $-m$ value (that is, a longer wavelength) results in a farther deflection in the $-y$ direction.

Using the method of stationary phase, we can evaluate the energy density distribution (24) as follows:

$$
\begin{aligned}
\bar{E}(x, y)=\frac{1}{2}\left|Q(t) m F(m)\left[2 \pi / \phi^{\prime \prime}(m)\right]^{1 / 2} e^{i \phi(m)+i \pi / 4}\right|^{2} \\
\sim d^{2} A_{0}^{2}\left(W H_{2}\right)^{-1}\left(\sigma_{f} / M\right)^{1 / 2}|F(m)|^{2}(-m)^{7 / 2}
\end{aligned}
$$

where $m$ satisfies the stationary phase equation (25). The result of (26) shows that the energy density $\bar{E}(x, y)$ is in proportion to $(-m)^{-7 / 2}$, suggesting that components with shorter wavelengths contribute more to the energy density over the shelf. The term $|F(m)|^{2}$, on the other hand, depends largely on the bottom friction.

In the limit of $E_{v}^{1 / 2} \rightarrow 0$, we find that $|G(m)| \leq 1$ and the amplitude envelope of $|F(m)|^{2}$ is equal to $\exp \left(-d^{2} m^{2} / 2\right)$. Solving $\partial \bar{E} / \partial m=0$ shows that the peak of the energy density envelope should be induced by the component with $m=$ $-d^{-1}(7 / 2)^{1 / 2}$. Nevertheless, for observed values of $d \sim 0.4$ and $1 / \sigma_{f}<O(150)$ this component, $m=-4.68$, falls out of the freely propagating Rossby wave range in the offshore ocean and consequently contributes little to the shelf/slope response (note that the cutoff wave number of the offshore Rossby wave is $m_{c}=\left[\alpha-\left(\alpha^{2}+\beta^{2}\right)^{1 / 2}\right] / 2 \sigma_{f}$; see Figure 11). In this case the shelf energy density peak is instead induced by the component which has the largest wave number in the freely propagating Rossby wave range, that is, $m=m_{c}$. Substituting $m=m_{c}$ into (25) and calculating its $y$ deflection, we obtain the energy density peak over the shelf at

$$
y_{c}--\left\{\frac{M}{2\left[\left(\alpha^{2}+\beta^{2}\right)^{1 / 2}-\alpha\right]}\right\}^{1 / 2}\left[1-\frac{\left(\alpha^{2}+\beta^{2}\right)^{1 / 2}-\alpha}{M}\right] W
$$

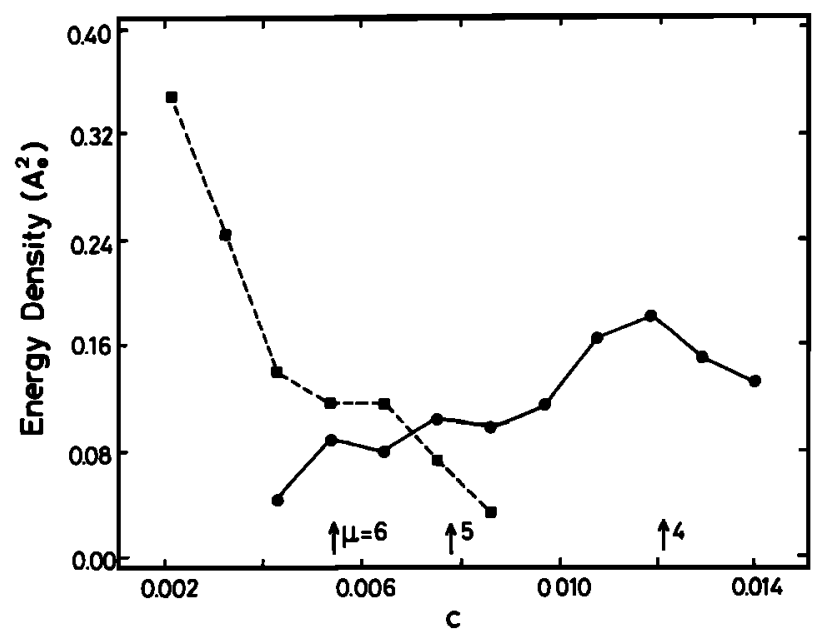

Fig. 7. Maximum energy density values at the shelf break induced by forcing sources with different propagating speeds $\left(E_{v}^{1 / 2}=\right.$ $0.0025)$. In the figure, circles indicate the maximum values caused by the bottom-trapped TRWs, while squares indicate those caused by the surface-intensified baroclinic waves. 

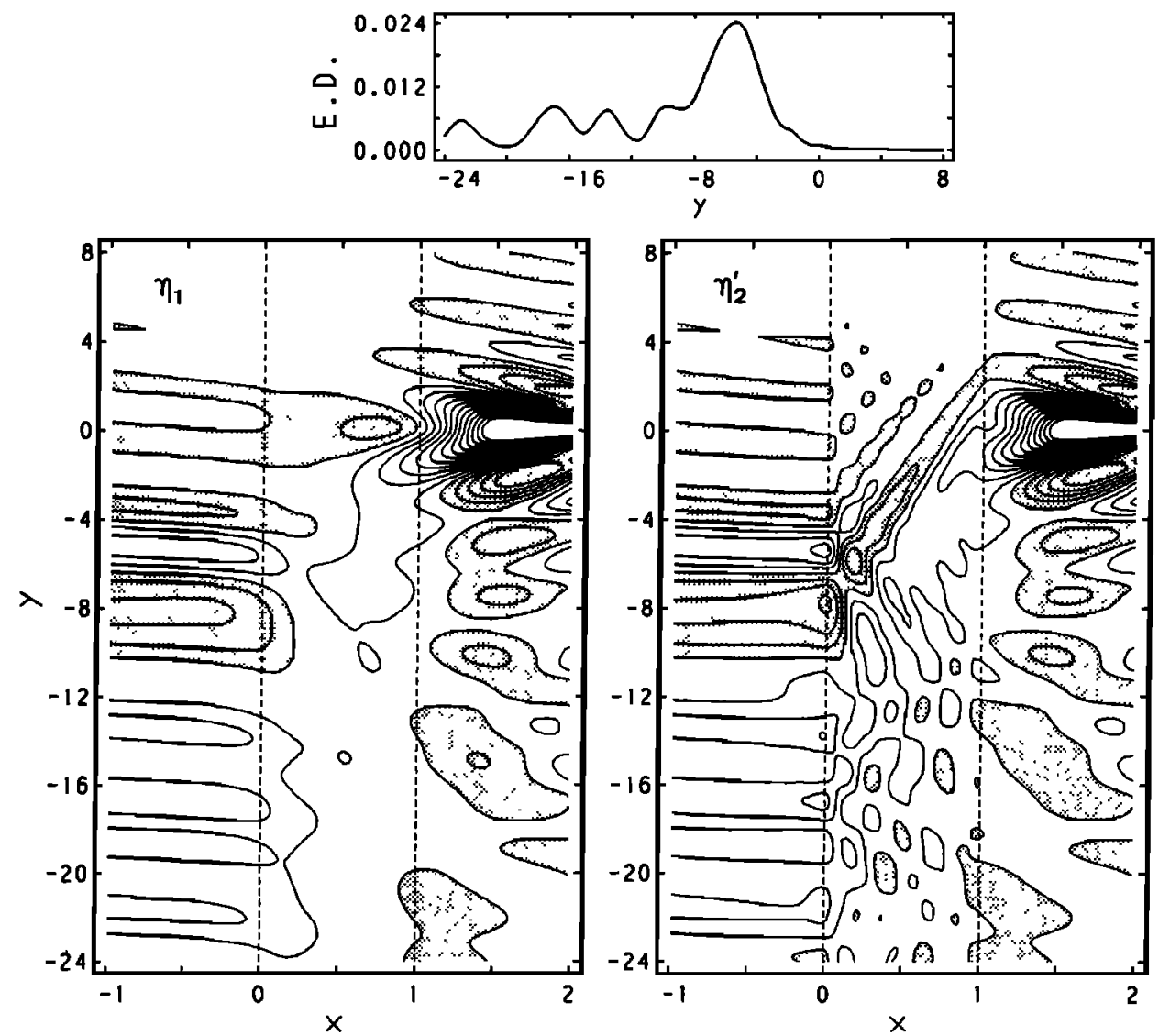

Fig. 8. The $\left(\eta_{1}, \eta_{2}^{\prime}\right)$ pattern and averaged shelf energy density distribution induced by a localized forcing source oscillating at $1 / \sigma_{f}=110$ when $E_{v}^{1 / 2} \rightarrow 0$. The contour interval values for $\eta_{1}$ and $\eta_{2}^{\prime}$ are $0.05 A_{0}$, where $A_{0}$ is the amplitude of the forcing source.

Notice that this $y_{c}$ value, as shown in Figure 10 by the dashed line, is independent of the $1 / \sigma_{f}$ values. This is the same tendency observed in the numerical calculations as $E_{v}^{1 / 2} \rightarrow 0$ (see solid circles in Figure 10). The physical explanation is that ray paths of the offshore cutoff wave components in the slope region deflect at the same angle regardless of the offshore forcing frequencies (see the dashed line in Figure 11). The difference between (27) and the solid circles in Figure 10 occurs because wave components with $m$ $=m_{c}$ do not necessarily have large transmission coefficients (Figure 3a).

The term $|F(m)|^{2}$ becomes a complicated function of $m$ when bottom friction is considered. Instead of solving $\partial \bar{E} / \partial m$ $=0$ in detail to determine the wave component causing the energy density peak over the shelf, we will next discuss qualitatively the frictional effect on this wave component. First, we recall that the shelf energy density $\breve{E}(x, y)$ is proportional to $(-m)^{7 / 2}$, indicating that wave components with larger wave numbers tend to make larger contributions. In the above inviscid case the energy density peak is in fact induced by the wave component that has the largest wave number in the offshore freely propagating Rossby wave range (i.e., the cutoff wave number $m=m_{c}$ ). It is worth noting that this $m_{c}$ value, as shown by the dashed line in Figure 11 , increases in proportion to $1 / \sigma_{f}$. Since wave components with larger wave numbers are more subject to damping by bottom friction (cf. Figure 6), it is clear that as the $1 / \sigma_{f}$ value increases, the wave component with $m=m_{c}$ will be strongly damped out and the shelf energy density peak will be produced by a wave component with a smaller wave number that survives the damping of bottom friction. From the result of Figure 11 we can thus expect the shelf energy density peak in the frictional cases to appear at a farther $-y$ location than the component with $m=m_{c}$ as the $1 / \sigma_{f}$ value increases. In fact, this $1 / \sigma_{f}$ dependence of the shelf energy density peak is the result obtained in the numerical calculations including bottom friction (open circles in Figure 10).

Finally, we plot in Figure 12 the shelf energy density peak values obtained in the frictional cases against the $1 / \sigma_{f}$ value. The fact that larger energy density peaks are induced in lower-frequency forcing cases is due to the increase in the number of offshore Rossby wave components that are able to transfer energy from the offshore forcing source to the slope region. Notice that the energy density peak values induced in the present oscillating forcing cases are generally smaller than those in the propagating forcing cases of section 3 (cf. Figure 7). A maximum energy density value of $0.018 A_{0}^{2}$, in the case of $1 / \sigma_{f}=110$, for example (Figure 9), indicates that the maximum velocity over the outer shelf is about $0.09 v_{m}$, where $v_{m}$ is the maximum velocity of the offshore forcing eddy. The energy density peak in the oscillating forcing cases is small because dispersion over the slope region occurs in both the $x$ and $y$ directions, whereas the propagating forcing cases, dispersion occurs in only the $x$ direction. 

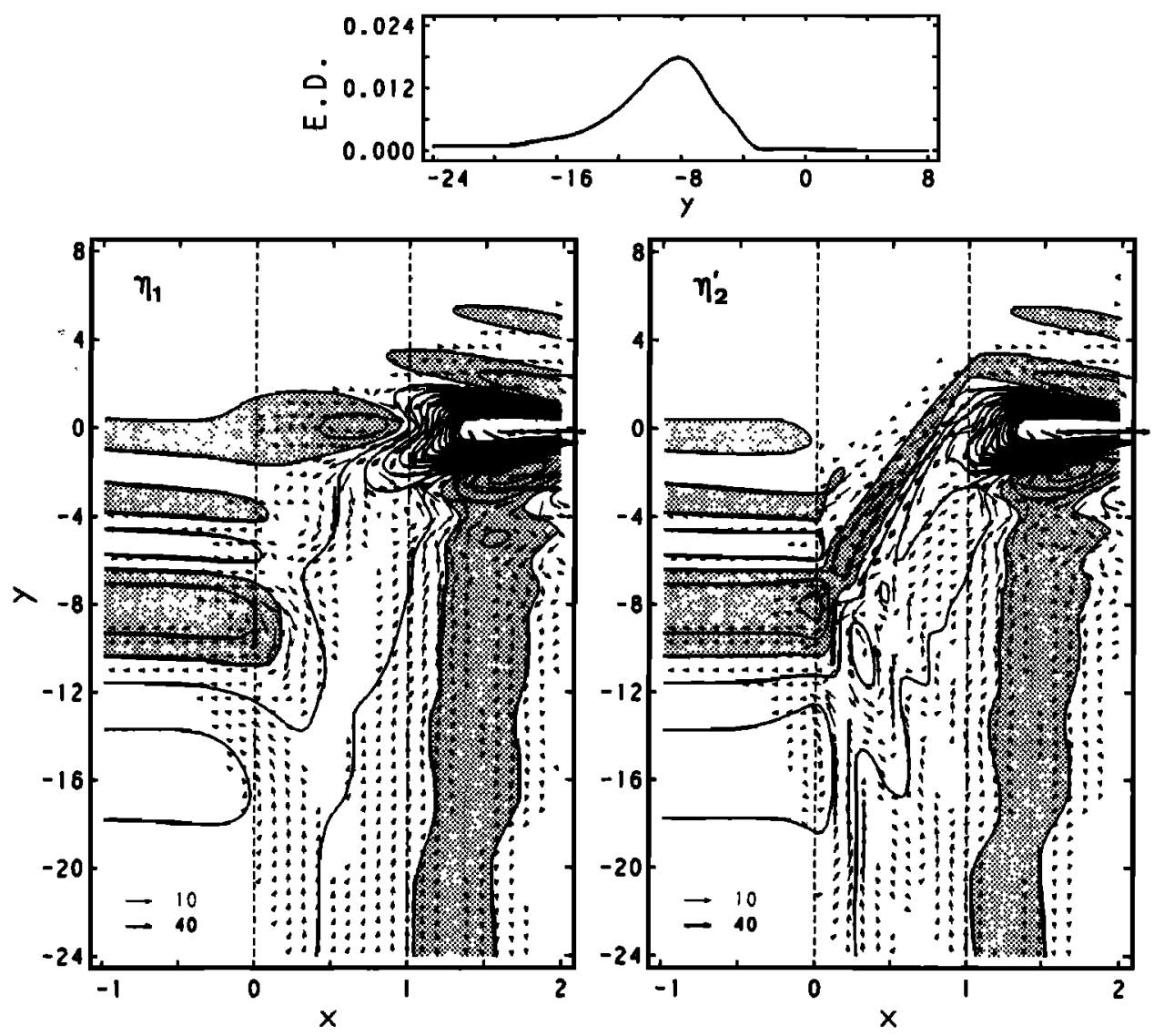

Fig. 9. As in Figure 8 except for the inclusion of bottom friction $\left(E_{v}^{1 / 2}=0.0025\right)$. The contour interval values for $\eta_{1}$ and $\eta_{2}^{\prime}$ are $0.0375 A_{0}$, and the unit for velocity vectors is $0.03 A_{0}$.

\section{Conclusions and Discussion}

Using a two-layer $\beta$ plane ocean model, we investigated the shelf/slope responses induced by localized forcing sources which (1) propagate in the along-slope direction and (2) have oscillating amplitudes. In the real ocean the first forcing situation corresponds to cutoff warm eddies propagating in the along-slope direction as observed by Joyce [1984]. The second forcing situation with a single oscillating frequency, on the other hand, can be regarded as one component in the frequency domain representing the stationary meandering of the Gulf Stream front as observed by both Luyten [1977] and Louis and Smith [1982]. In this section we will summarize the results obtained in the present study and discuss the applicability of these results to real ocean situations. For easy comparison the variables will be expressed in their dimensional units.

We found that when the offshore forcing source propagates in the along-slope direction with the shelf to the right ( $c$ $<0$ ), the shelf/slope responses are dominated by the bottomtrapped TRWs in the inviscid limit and their intensity depends strongly on the value

$$
\mu=\frac{1}{\pi}\left(-f_{0} M / c\right)^{1 / 2}\left[W+\frac{f_{0} c}{g^{\prime} H_{1} M^{2}}\left(\frac{H_{1}-H_{2}}{H_{1}}\right)\right]
$$

Maximum responses occur when the $\mu$ value is close to an integer, and the responses are minimum when $\mu$ is an integer plus $1 / 2$. The underlying physics for this $\mu$ dependence is as follows: Because of the dominance of the vortex stretching term in the vorticity balance in the lower layer slope region, a propagating forcing source in the offshore ocean tends to induce wave components over the slope region that have a similar cross-slope wave structure. When the propagating speed $c$ is such that the resultant phase change across the

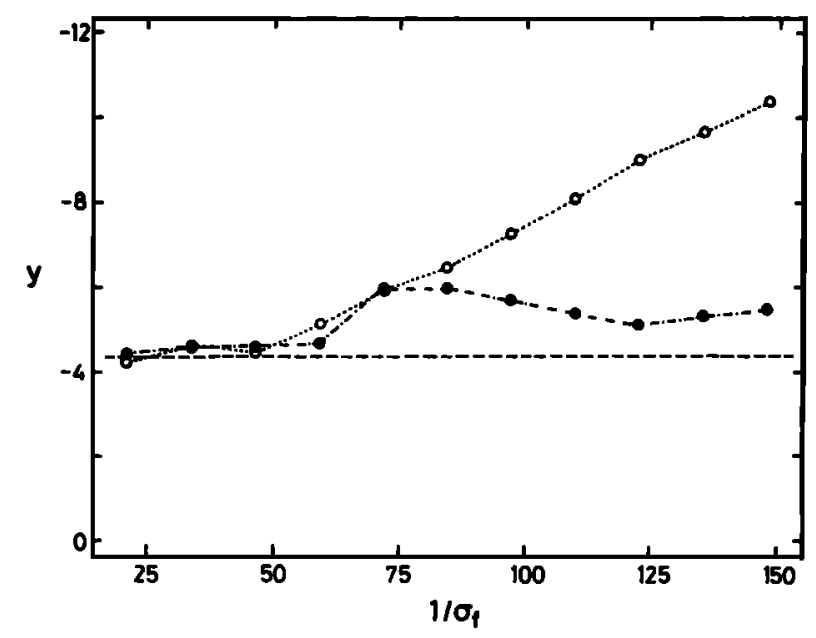

Fig. 10. Locations at the shelf break of the energy density peak induced by forcing sources oscillating in amplitude. Solid circles show the numerical results obtained in the inviscid limit, while open circles show those obtained when $E_{v}^{1 / 2}=0.0025$. The dashed line shows the location deflected by the offshore cutoff Rossby wave component (see (27)). 
slope region is close to $n \pi$ (that is, when $\mu$ is close to the integer $n$ ), all wave components can reach the outer shelf without much energy reflection. This consequently causes strong responses in the shelf/slope region.

With the inclusion of bottom friction we found that this $\mu$ dependence of the shelf/slope response is valid only for the fast-propagating forcing cases, wherein the wave components have large cross-slope length scales and are weakly influenced by the bottom friction. When the propagating speed of the source is slow, however, the excited small-scale bottom-trapped TRWs are effectively damped out by the bottom friction. As a result, shelf energy density maxima in the slow-propagating forcing cases are mainly determined by the surface-intensified Rossby waves, as opposed to the fast-propagating forcing cases where the energy density maxima are determined by the bottom-trapped TRWs (Figure 7).

In ocean regions north of the Gulf Stream beyond Cape Hatteras, warm-core rings are frequently observed to move west-southwestward with speeds of $3 \sim 5 \mathrm{~cm} \mathrm{~s}^{-1}$ [Joyce, 1984]. An estimation for $M=O\left(0.04 \mathrm{~km}^{-1}\right), W=O(60 \mathrm{~km})$, $f_{0}=10^{-4} \mathrm{~s}^{-1}, g^{\prime} H_{1}=O\left(4 \times 10^{4} \mathrm{~cm}^{2} \mathrm{~s}^{-2}\right)$, and $H_{1} \ll H_{2}$ leads to $\mu=5.1 \sim 6.7$. From the result of Figure 7 we notice that these $\mu$ values fall in the parameter range where the surface and bottom wave components are both important to the shelf/slope responses. Evidence showing the existence of long-period surface-intensified current fluctuations has been observed by Louis et al. [1982] at the continental margin off Novia Scotia. They attributed these fluctuations to the baroclinic radiation field associated with the offshore eddies.

For the case of $c=-0.0055$ (Figure 5), detailed study of velocity components showed that a jet moving in the $+y$ direction appeared near the shelf break at $y-c t=-2.7 \sim$ $\mathbf{- 0 . 9}$. The jet's location and direction are similar to those of the shelf break jet found by Chapman and Brink [1987]. It should be noticed, however, that the shelf break jet in the Chapman and Brink model is directly driven by the forcing source when it is located close to the shelf break or when the stratification is increased, allowing the forcing eddy to reach the shelf break before decaying. Since the forcing source in the present study exists in the offshore region, the jet occurs

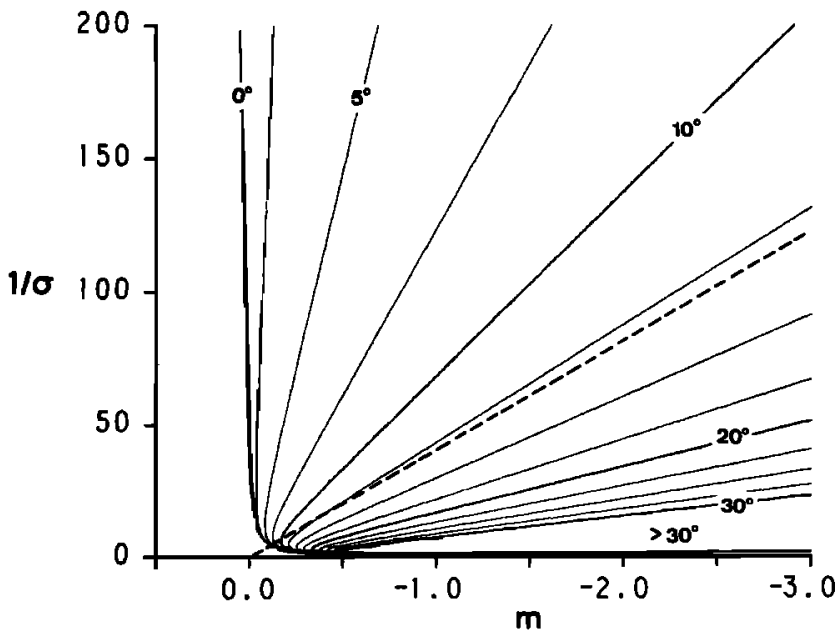

Fig. 11. Deflected angles between the ray path and the $-y$ axis of the TRWs in the slope region. The dashed line indicates the cutoff wave number for the offshore barotropic Rossby waves.

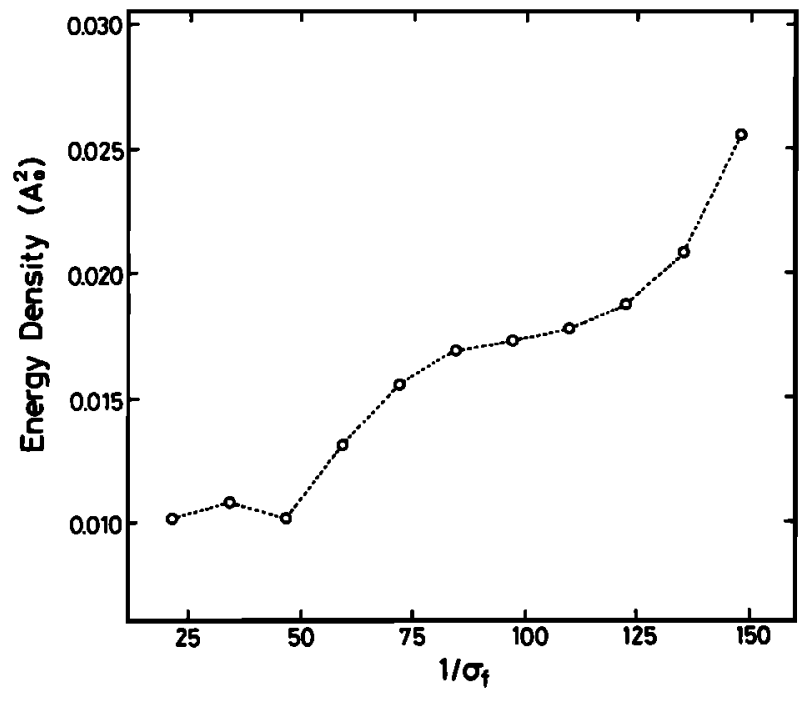

Fig. 12. Maximum energy density values at the shelf break induced by forcing sources oscillating in amplitude $\left(E_{\nu}^{1 / 2}=0.0025\right)$.

after bottom-trapped TRW components have weakly dispersed and interfered with their reflected waves near the shelf break. As a result, the direction and the location of this jet depend largely on the propagating speed $c$ : when $c=$ -0.0080 , for example, the jet moves in the $-y$ direction around the location of $y-c t=-2.7 \sim-0.9$ (not shown).

Unlike in the Chapman and Brink's model, the present study shows that a propagating forcing source located away from the shelf break can still be significant for the neighboring shelf/slope responses. The difference between the present model and that of Chapman and Brink stems from the inclusion of the planetary $\beta$ effect. The planetary $\beta$ in the offshore regions supports the barotropic Rossby wave motions, enabling the energy of the offshore forcing source to be transferred to the slope region. Moreover, the planetary $\beta$ supports the surface-intensified wave motions, which, as we found in the present study, have a direct influence on the shelf/slope responses.

When the localized offshore forcing source is stationary and has an oscillating amplitude, we found that maximum responses occur along a confined line area over the $-y$ slope region. Using the method of stationary phase, we found that in the inviscid limit, this line area can be approximated by

$y \sim-\left[\frac{f_{0} M}{2 \beta_{0}(1+\sin \theta)}\right]^{1 / 2}\left[1-\frac{\beta_{0}(1+\sin \theta)}{f_{0} M}\right](W-x)$

where $x$ is the offshore distance from the shelf break. Physically, (29) expresses the ray path of the cutoff wave component of the offshore barotropic Rossby wave. Notice that the $y$ value in (29) is independent of $\sigma_{f}$, the oscillating frequency of the forcing source, but decreases when the bathymetry is more zonal $(\theta \rightarrow \pi / 2)$. Typical values of $M f_{0}=$ $O\left(4 \times 10^{-11} \mathrm{~cm} \mathrm{~s}^{-1}\right), W=O(60 \mathrm{~km})$, and $\theta=\pi / 4 \sim \pi / 2$ yield $y=420 \sim 460 \mathrm{~km}$ at the shelf break $(x=0)$.

By including the bottom friction we found that although the energy density peaks appear at around the same $-y$ location as in the inviscid case, they tend to shift to a farther $-y$ location (or shift to an outer slope location along a fixed cross-slope line) as the oscillating period of the forcing 
source increases. This shift occurs because bottom friction is more effective in damping out small-scale wave components with small $-y$ deflections when $1 / \sigma_{f}$ becomes large. Because of the increase of the incident Rossby wave components permissible in the offshore ocean, we further found that the energy density peak value in the shelf/slope region tends to increase as the forcing period increases.

From moored results of current and temperature across the continental rise south of Cape Cod, Hogg [1981] observed that the energy maxima for the three different period bands appear further north with decreasing period and that the maximum energy values increase along with the period (his Figure 8). Although the present model is too simple to do quantitative comparisons, these observational results are consistent with and can qualitatively be explained by the result of the present study.

Finally, it is worth emphasizing that although the offshore current meandering and warm-core eddies are governed by nonlinear dynamics, their influence upon the neighboring field is mainly via the forcing of the dispersive Rossby waves [e.g., McWilliams and Flierl, 1979]. Shelf/slope responses induced by such offshore forcing sources can be adequately understood by using our present linear model. When the localized forcing source is located close to the shelf break, as observed along the southeast U.S. outer continental shelf [e.g., Lee and Atkinson, 1983] and the East China Sea continental shelf [Sugimoto et al., 1988], the nonlinear effects of the forcing source on the shelf/slope dynamics should no longer be neglected. The nonlinear effect can also enter the problem when the forcing source is close to the shelf/slope region, causing resonance in the response field. These nonlinear effects will be incorporated in our subsequent studies on shelf/slope responses.

\section{APPENDIX}

The intrinsic phase change of TRWs across a slope region has been shown by Kroll and Niiler [1976] and $\mathrm{Ou}$ and Beardsley [1980] to be a crucial quantity in determining the energy transmission by monochromatic wave forcings. In a two-layer ocean the intrinsic phase change of bottomtrapped TRWs can be evaluated as follows.

When the forcing period is short such that surfaceintensified waves are not induced (in Figure 3: $1 / \sigma<180$ ), we can neglect the influence from the upper layer motion and simplify the lower layer equation (10) to

$$
\begin{aligned}
\varepsilon Z_{2 x x}^{\prime}+ & (i \beta / \sigma+M) Z_{2 x}^{\prime} \\
& +\left[-\varepsilon m^{2}+(\alpha-M) m / \sigma-1 / S H(x)\right] Z_{2}^{\prime}=0
\end{aligned}
$$

Assuming $\Delta \equiv E_{v}^{1 / 2} / 2 \sigma \ll 1$ and transforming $Z_{2}^{\prime}=Y_{2}^{\prime} \exp$ $[-(i \beta / \sigma+M) x / 2 \varepsilon]$, we can rewrite (A1), to $O\left(\Delta^{2}\right)$, as

$$
\begin{aligned}
Y_{2 x x}^{\prime}+\left\{K_{s}^{2}-\right. & 1 / S H(x)-\Delta i[\alpha m / \sigma-M m / \sigma \\
& \left.\left.+\beta^{2} / 2 \sigma^{2}-M^{2} / 2-1 / S H(x)\right]+O\left(\Delta^{2}\right)\right\} Y_{2}^{\prime}=0
\end{aligned}
$$

where

$$
K_{s}^{2} \equiv \beta^{2} / 4 \sigma^{2}+(\alpha-M) m / \sigma-m^{2}-M^{2} / 4
$$

satisfies the dispersion relation for the nondivergent barotropic TRWs in the slope region. Notice that the term $-m M / \sigma$ in (A2) generally predominates over other terms. When the slope parameter $M \sim O(1)$, as in the case under consideration, we have $-m M / \sigma \gg 1 / S H(x)$ with the exception of $m / \sigma$ $\rightarrow 0$. Physically, this means that the effect of vortex stretching caused by the slope inclination predominates over that caused by the interfacial elevations. This fact enables us to obtain the WKB solution for (A1):

$$
Z^{\prime}(x) \propto \exp \left\{ \pm i \int_{0}^{x}\left[K^{2}-1 / S H(x)+O\left(\Delta^{2}\right)\right]^{1 / 2} d x\right\}
$$

Evaluating the integrand in (A3) at $x=W$ and noting that $-m M / \sigma \gg 1 / S H$, we obtain the intrinsic phase change across the slope as

$$
\begin{array}{r}
I \equiv \int_{0}^{W}\left[K_{s}^{2}-e^{-M x} / S H_{2}+O\left(\Delta^{2}\right)\right]^{1 / 2} d x \rightarrow K_{s} W \\
-\left(e^{M W}-1\right) / M S K_{s}+O\left(\Delta^{2}\right)
\end{array}
$$

If $S$ is a priori set to be infinite and $\Delta \rightarrow 0$, (A4) reduces to the result obtained by Kroll and Niiler [1976] for the nondivergent barotropic system. Notice that inclusion of bottom friction only influences the intrinsic phase change to $O\left(\Delta^{2}\right)$. Also notice that the solution (A4) fails in the parameter range of $m / \sigma \rightarrow 0$, thus causing the discrepancy between the results of maximum FTCs and $I=\pi$ in Figure 3 .

When the forcing period is large, allowing propagating baroclinic wave modes, the intrinsic phase change of bottom-trapped TRWs is still given by (A4). This is because the coupling between the surface waves and the bottom-trapped TRWs is generally negligible in the slope region [Suginohara, 1981]. Detailed discussion, however, will be omitted from the present paper.

Acknowledgments. I would like to thank N. Imasato for suggesting I research the interesting subject of shelf/slope responses to the localized offshore forcing and for critically reading the manuscript. I am also grateful to S. Imawaki, A. Kubokawa, P. P. Niiler, and S. Saki for their discussions in the course of the study. Thanks are also extended to the two reviewers for their comments. The computations were performed on the FACOM-M780 and VP-200 computer at the Data Processing Center of Kyoto University.

\section{REFERENCES}

Allen, J. S., A simple model for stratified shelf flow fields with bottom friction, J. Phys. Oceanogr., 14, 1200-1214, 1984.

Chapman, D. C., and K. H. Brink, Shelf and slope circulation induced by fluctuating offshore forcing, J. Geophys. Res., 92, $11,741-11,759,1987$.

Csanady, G. T., The arrested topographic wave, J. Phys. Oceanogr., 8, 47-62, 1978.

Gratton, Y., and P. H. LeBlond, Vorticity waves over strong topography, J. Phys. Oceanogr., 16, 151-166, 1986.

Hogg, N., Topographic waves along $70^{\circ} \mathrm{W}$ on the continental rise, $J$. Mar. Res., 39, 627-649, 1981.

Joyce, T. M., Velocity and hydrographic structure of a Gulf Stream warm-core ring, J. Phys. Oceanogr., 14, 936-947, 1984.

Kroll, J., The kinetic energy on a continental shelf from topographic Rossby waves generated off the shelf, J. Phys. Oceanogr., 9, $712-723,1979$.

Kroll, J., and P. P. Niiler, The transmission and decay of barotropic topographic Rossby waves incident on a continental shelf, $J$. Phys. Oceanogr., 6, 432-450, 1976.

LeBlond, P. H., and L. A. Mysak, Waves in the Ocean, 602 pp., Elsevier, New York, 1978. 
Lee, T. N., and L. P. Atkinson, Low-frequency current and temperature variability from Gulf Stream frontal eddies and atmospheric forcing along the southern $U$. S. outer continental shelf, J. Geophys. Res., 88, 4541-4567, 1983.

Louis, J., and P. C. Smith, The development of the barotropic radiation field of an eddy over a slope, J. Phys. Oceanogr., 12, 56-73, 1982.

Louis, J., B. Petrie, and P. Smith, Observations of topographic Rossby waves on the continental margin off Nova Scotia, J. Phys. Oceanogr., 12, 47-55, 1982.

Luyten, J. R., Scales of motion in the Gulf Stream and across the continental rise, J. Mar. Res., 35, 49-74, 1977.

McWilliams, J. C., and G. R. Flierl, On the evolution of isolated nonlinear vortices, J. Phys. Oceanogr., 9, 1155-1182, 1979.

Ou, H. W., and R. C. Beardsley, On the propagation of free topographic Rossby waves near continental margins, 2, Numerical model, J. Phys. Oceanogr., 10, 1323-1339, 1980.

Pedlosky, J., Geophysical Fluid Dynamics, 624 pp., SpringerVerlag, New York, 1979.

Rhines, P. B., Slow oscillations in an ocean of varying depth, 1, Abrupt topography, J. Fluid Mech., 37, 161-189, 1969.
Shaw, P. T., and C. Y. Peng, A numerical study of the propagation of topographic Rossby waves, J. Phys. Oceanogr., 17, 358-366, 1987.

Smith, P. C., Eddies and coastal interactions, in Eddies in Marine Science, edited by A. R. Robinson, pp. 446-480, Springer-Verlag, New York, 1983

Sugimoto, T., S. Kimura, and K. Miyaji, Meander of the Kuroshio front and current variability in the East China Sea, J. Oceanogr. Soc. Jpn., 44, 125-135, 1988.

Suginohara, N., Quasi-geostrophic waves in a stratified ocean with bottom topography, J. Phys. Oceanogr., 11, 107-115, 1981.

Thompson, R. O. R. Y., Topographic Rossby waves at a site north of the Gulf Stream, Deep Sea Res., 18, 1-20, 1971.

B. Qiu, Department of Physical Oceanography, Woods Hole Oceanographic Institution, Woods Hole, MA 02543.

(Received June 12, 1989:

revised September 28, 1989,

accepted October 24,1989 .) 